\title{
Burkholderia gladioli - a predictor of poor outcome in cystic fibrosis patients who receive lung transplants? A case of locally invasive rhinosinusitis and persistent bacteremia in a 36-year-old lung transplant recipient with cystic fibrosis
}

\author{
Bradley S Quon MD FRCPC 1 , James D Reid MD ${ }^{1}$, Patrick Wong BSc ${ }^{1}$, Pearce G Wilcox MD FRCPC ${ }^{1}$, \\ Amin Javer MD FRCSC ${ }^{2}$, Jennifer M Wilson MD FRCPC ${ }^{1,3}$, Robert D Levy MD FRCPC FCCP1,3
}

BS Quon, JD Reid, P Wong, et al. Burkholderia gladioli - a predictor of poor outcome in cystic fibrosis patients who receive lung transplants? A case of locally invasive rhinosinusitis and persistent bacteremia in a 36-year-old lung transplant recipient with cystic fibrosis. Can Respir J 2011;18(4):e64-e65.

There have been very few reports describing postlung transplant outcomes in patients' infected/colonized with Burkholderia gladioli pretransplant. A case involving a lung transplant recipient with cystic fibrosis who ultimately died as a result of severe rhinosinusitis due to $B$ gladioli infection in the context of postlung transplant immunosuppression is reported.

Key Words: Bacteremia; Case report; Cystic fibrosis; Lung transplant; Opportunistic infection

$\mathbf{B}$ urkholderia gladioli are ubiquitous Gram-negative bacteria that are able B to occupy diverse environments including soil, plants and the respiratory tract of humans (1). Although B gladioli have traditionally been recognized as plant pathogens, they are increasingly being reported to cause infections in humans. They are closely related to Burkholderia cepacia complex, which are well known for their ability to cause severe illness in cystic fibrosis patients. Based on poor postlung transplant outcomes, prelung transplant infection/colonization with B cepacia complex is currently an absolute contraindication for lung transplantation at many lung transplant centres in North America (2).

\section{CASE PRESENTATION}

A 36-year-old woman with advanced cystic fibrosis (CF) lung disA ease underwent bilateral lung transplantation in 2007. Her pretransplant history was notable for repeated pulmonary exacerbations involving methicillin-resistant Straphylococcus aureus (MRSA) and Burkholderia gladioli, but without clinically significant sinus disease requiring investigations and/or treatment. Ten months post-transplant, she presented with a right frontal headache. A computed tomography scan revealed severe paranasal sinus disease and she was diagnosed with severe rhinosinusitis. She required extensive sinus surgery, and sinus cultures demonstrated heavy growth of MRSA and scant growth of B gladioli. She was initially treated with intravenous and topical vancomycin. Her immunosuppressive regimen was modified with a reduction in her maintenance prednisone dose and continuation of both tacrolimus and azathioprine. Her initial postoperative course was promising; however, three weeks later, she developed fever, recurrent sinus symptoms and her blood cultures grew B gladioli, which demonstrated in vitro susceptibility to all antibiotics tested including amikacin, tobramycin, meropenem, piperacillin-tazobactam, ciprofloxacin
Le Burkholderia gladioli, un prédicteur de mauvaise issue chez les patients atteints de fibrose kystique qui subissent une greffe des poumons? Un cas de rhinosinusite avec envahissement local et de bactériémie persistante chez un greffé de 36 ans atteint de fibrose kystique

\begin{abstract}
Après une greffe pulmonaire, très peu de rapports décrivent les issues des patients qui étaient infectés ou colonisés par le Burkholderia gladioli avant la greffe. Est exposé le cas d'un greffé des poumons atteint de fibrose kystique qui est finalement décédé à cause d'une grave rhinosinusite causée par une infection à $B$ gladioli dans le contexte de l'immunosuppression après la greffe pulmonaire.
\end{abstract}

and trimethoprim-sulfamethoxazole. She underwent additional sinus surgery that did not improve her symptoms. Sinus cultures at the time of her second sinus surgery demonstrated light growth of both MRSA and B gladioli. She was placed on intravenous vancomycin, meropenem, ceftazadime, oral trimethoprim-sulfamethoxazole and topical gentamicin. Although she initially improved following this treatment, she went on to develop bilateral purulent otitis media, mastoiditis and petrous bone apicitis (Figure 1). Subsequent sinus cultures demonstrated heavy growth of $B$ gladioli. Her overall clinical condition progressively deteriorated, and she developed persistent $B$ gladioli bacteremia, which developed resistance to all available antibiotics except piperacillin-tazobactam. Unfortunately, despite six months of continuous broad-spectrum intravenous antibiotics and six sinus surgeries, she eventually succumbed to the infection and died 18 months following lung transplantation.

\section{DISCUSSION}

We contribute to a growing body of evidence that $B$ gladioli infection in CF patients prelung transplant can result in significant posttransplant morbidity and mortality (3-5). Our described case of a CF patient with chronic respiratory infection involving $B$ gladioli prelung transplant tragically resulted in locally invasive sinus disease, persistent bacteremia and death 18 months post-transplant. Although MRSA may have contributed initially to her sinus disease, $B$ gladioli became the primary pathogen because her clinical deterioration correlated closely with heavy growth of $B$ gladioli from the sinuses and subsequent bacteremia. Her sinus infection was exceedingly difficult to control despite continuous intravenous antibiotics in the face of underlying immunosuppression, challenges achieving local source control and

${ }^{1}$ Department of Medicine; ${ }^{2}$ Department of Surgery, University of British Columbia; ${ }^{3}$ British Columbia Transplant, Vancouver,

British Columbia

Correspondence: Dr Robert D Levy, Divsion of Respiratory Medicine, Department of Medicine, St Paul's Hospital, Providence Health,

8B-1081 Burrard Street, Vancouver, British Columbia V6Z 1Y6. Telephone 604-806-8818, fax 604-806-8839,

e-mail RLevy@providencehealth.bc.ca 
eventual development of antibiotic resistance. Interestingly, she did not grow B gladioli in her transplanted lungs and did not demonstrate evidence of allograft dysfunction based on symptoms, spirometry or chest imaging. The present case report follows a recently documented case of a CF lung transplant recipient with a mediastinal abscess due to B gladioli diagnosed postlung transplant that was also difficult to control but eventually responded to a prolonged course of intravenous antibiotics and surgical debridement (3).

Although there is general consensus of poor post-transplant outcomes in CF patients harbouring Burkholderia cepacia complex (Bcc) (2), experience with B gladioli infection is limited. Several distinct species constitute what is now referred to as Bcc, with apparent considerable variation with respect to their virulence in CF patients. Based on small studies, some centres have revised their lung transplant eligibility criteria to exclude B cenocepacia-infected patients, while offering transplant to patients harbouring other Bcc genomovars (5). Although B gladioli are phenotypically very similar to genomovars of Bcc, they are molecularly distinct and, therefore, have been classified as an independent species due to the availability of advanced molecular identification techniques (1). Studies have suggested more transient infection, less antibiotic resistance and absence of patientto-patient transmission with B gladioli compared with Bcc (1).

Kennedy et al (4) conducted a review investigating postlung transplant outcomes in CF patients' infected/colonized with B gladioli, summarizing three new cases and four previous cases from the literature. One of seven patients died as a direct result of B gladioli infection; however, that patient exhibited evidence of disseminated disease before lung transplant. The remaining six patients responded favourably to antibiotic therapy post-transplant. In light of these findings, the authors concluded that caution should be exercised, but that pretransplant infection with $B$ gladioli should not contraindicate lung transplantation in patients with CF. A more recent retrospective review by Murray et al (5), utilizing data from the United States CF Foundation Patient Registry, demonstrated greater post-transplant mortality in $14 \mathrm{CF}$ lung transplant recipients infected with B gladioli compared with noninfected recipients, with an HR of $2.23(\mathrm{P}=0.04)$.

The present case adds to the growing body of evidence characterizing considerable morbidity and mortality related to opportunistic $B$ gladioli infection in CF lung transplant recipients. This information suggests that the identification of B gladioli in pretransplant CF patients warrants reconsideration as a possible important relative contraindication to lung transplantation, similar to Bcc.

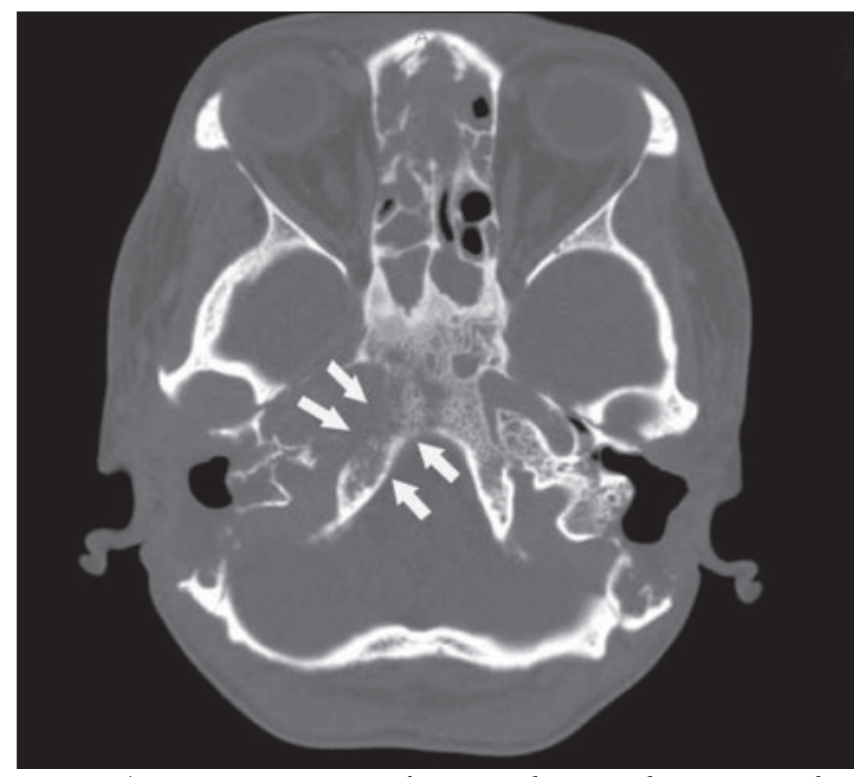

Figure 1) Extensive sinus opacification and petrous bone osteomyelitis caused by Burkholderia gladioli and methicillin-resistant Staphylococcus aureus infection in a lung transplant recipient with cystic fibrosis. The solid white arrows indicate the moth-eaten petrous aspect of the right temporal bone

\section{REFERENCES}

1. Segonds C, Clavel-Batut P, Thouverez M, et al. Microbiological and epidemiological features of clinical respiratory isolates of Burkholderia gladioli. J Clin Micro 2009;47:1510-6.

2. Chaparro C, Maurer J, Gutierrez C, et al. Infection with Burkholderia cepacia in cystic fibrosis: Outcome following lung transplantation. Am J Respir Crit Care Med 2001;163:43-8.

3. Church A, Sivasothy P, Parmer J, Foweraker J. Mediastinal abscess after lung transplantation secondary to Burkholderia gladioli infection. J Heart and Lung Transplant 2009;28:511-4.

4. Kennedy M, Coakley R, Donaldson S, et al. Burkholideria gladioli: Five year experience in cystic fibrosis and lung transplantation center. J Cystic Fibrosis 2007;6:267-73.

5. Murray S, Charbeneau J, Marshall BC, LiPuma JJ. Impact of Burkholderia infection on lung transplantation in cystic fibrosis. Am J Respir Crit Care Med 2008;178:363-71. 


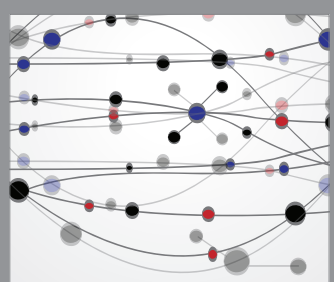

The Scientific World Journal
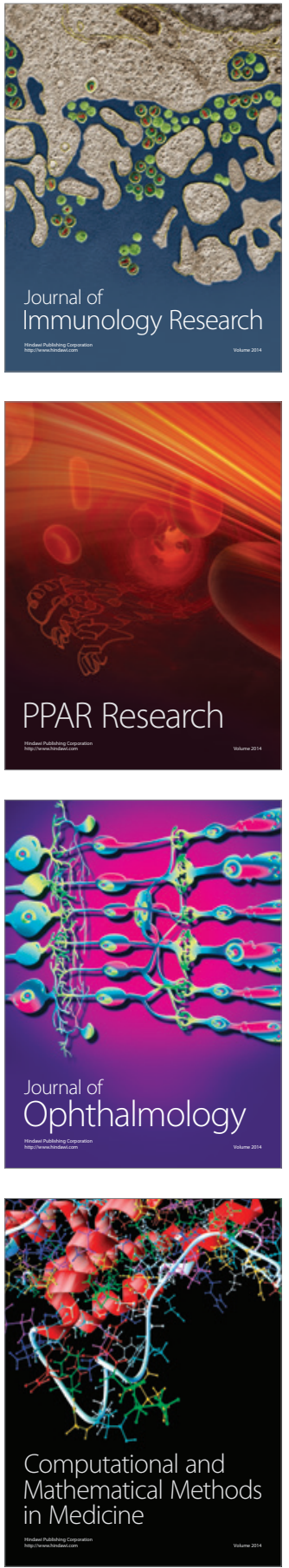

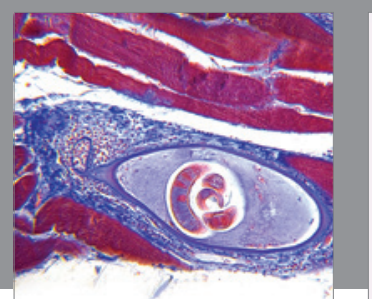

Gastroenterology Research and Practice

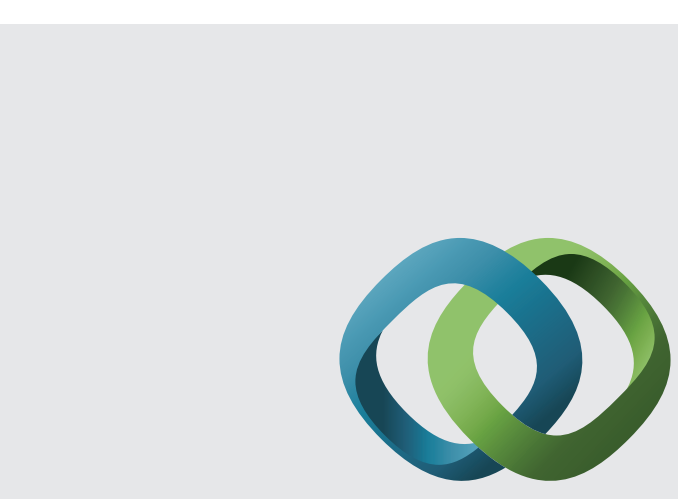

\section{Hindawi}

Submit your manuscripts at

http://www.hindawi.com
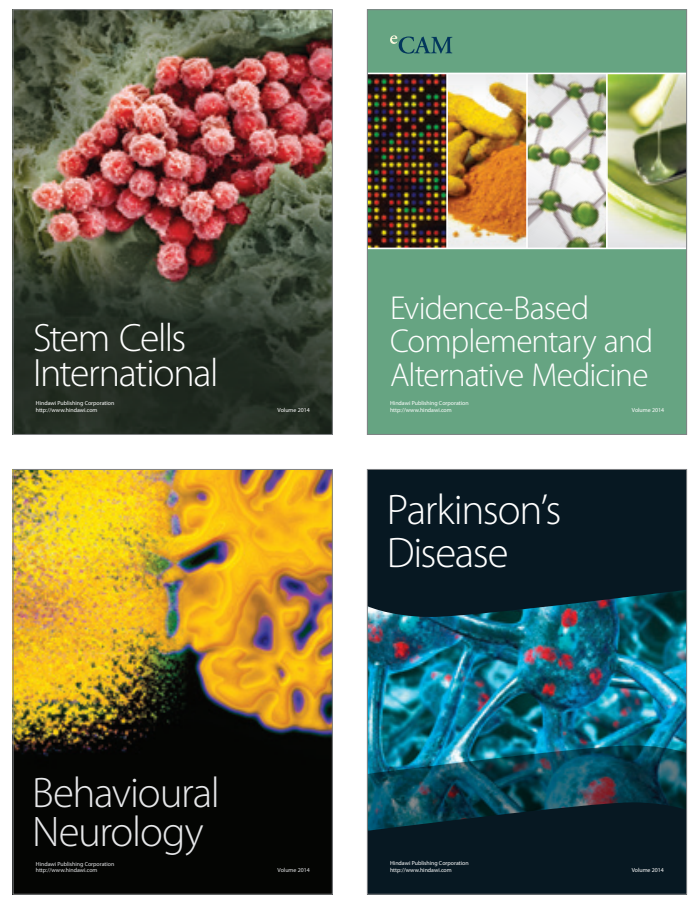
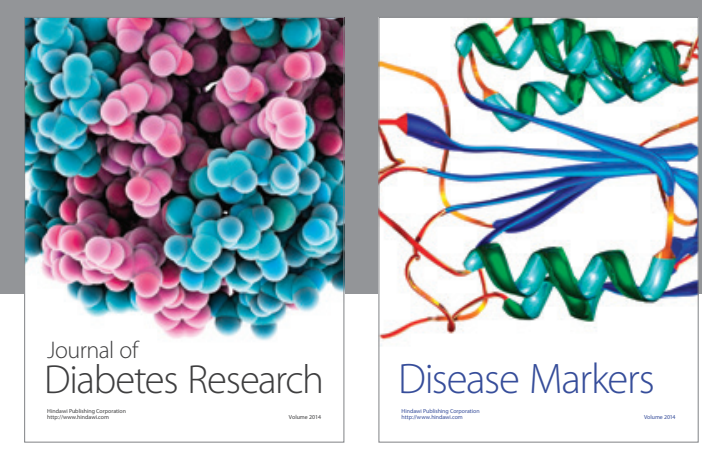

Disease Markers
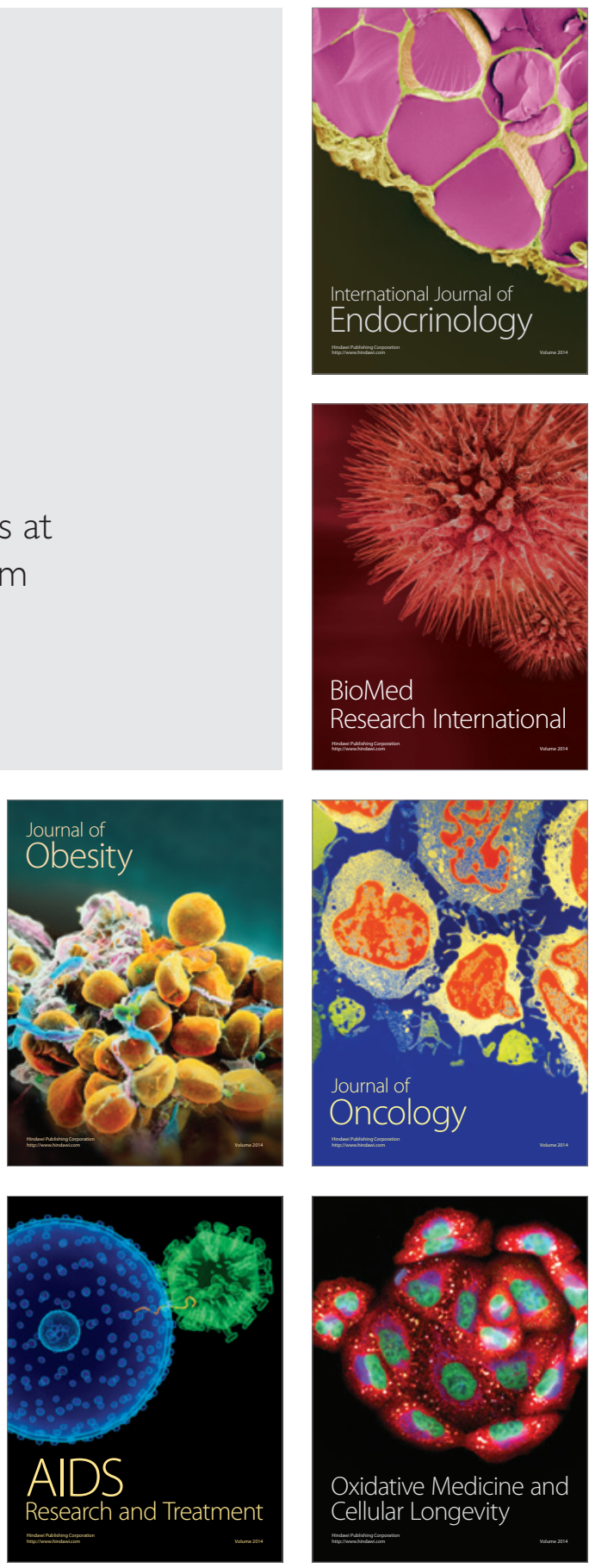